\title{
NEVANLINNA-PICK SPACES WITH HYPONORMAL MULTIPLICATION OPERATORS
}

\author{
MICHAEL HARTZ
}

\begin{abstract}
We show that the Hardy space on the unit disk is the only non-trivial irreducible reproducing kernel Hilbert space which satisfies the complete Nevanlinna-Pick property and hyponormality of all multiplication operators.
\end{abstract}

\section{INTRODUCTION}

Let $\mathcal{H}$ be a reproducing kernel Hilbert space on a set $X$ with kernel $K$. In this short note, we study the relationship between two possible properties of $\mathcal{H}$ : the complete Nevanlinna-Pick property and hyponormality of multiplication operators. Recall that $\mathcal{H}$ is said to be a Nevanlinna-Pick space if, given $z_{1}, \ldots, z_{n} \in X$ and $w_{1}, \ldots, w_{n} \in \mathbb{C}$, positivity of the matrix

$$
\left(\left(1-w_{i} \overline{w_{j}}\right) K\left(z_{i}, z_{j}\right)\right)_{i, j=1}^{n}
$$

is not only a necessary, but also a sufficient condition for the existence of a multiplier $\varphi$ on $\mathcal{H}$ of norm at most 1 with

$$
\varphi\left(z_{i}\right)=w_{i} \quad(i=1, \ldots, n) .
$$

If the analogous result for matrix-valued interpolation holds, then $\mathcal{H}$ is called a complete Nevanlinna-Pick space (compare Chapter 5 in [2]). Spaces with this property have attracted a lot of attention, and it is known that they admit appropriate versions of some classical theorems for the Hardy space $H^{2}$ on the disk, such as the commutant lifting theorem [5] (see also [3]), the Toeplitz-corona theorem [2, Section 8.4] and Beurling's theorem [2, Section 8.5].

The second property we consider is hyponormality of multiplication operators, that is, the property that for every multiplier $\varphi$ on $\mathcal{H}$, the corresponding multiplication operator $M_{\varphi} \in \mathcal{B}(\mathcal{H})$ is hyponormal. While multiplication operators are not normal in typical examples, they are subnormal and hence hyponormal for a number of reproducing kernel Hilbert spaces, including Hardy and Bergman spaces on domains in $\mathbb{C}^{d}$.

Two results concerning weighted Hardy spaces serve as a motivation for the study of the relationship between the two properties. Suppose for a

2010 Mathematics Subject Classification. Primary 46E22; Secondary 47B32, 47B20.

Key words and phrases. Reproducing kernel Hilbert spaces, Nevanlinna-Pick kernels.

The author is partially supported by an Ontario Trillium Scholarship. 
moment that $\mathcal{H}$ is a reproducing kernel Hilbert space on the open unit disk $\mathbb{D}$ with kernel $K$ of the form

$$
K(z, w)=\sum_{n=0}^{\infty} a_{n}(z \bar{w})^{n} \quad(z, w \in \mathbb{D}),
$$

where $\left(a_{n}\right)$ is a sequence of positive numbers with $a_{0}=1$. Note that the classical Hardy space $H^{2}$ corresponds to the choice $a_{n}=1$ for all $n$, in which case we recover the Szegó kernel $(1-z \bar{w})^{-1}$. We assume that multiplication by the coordinate function $z$ induces a bounded multiplication operator $M_{z}$ on $\mathcal{H}$. Equivalently, the sequence $\left(a_{n} / a_{n+1}\right)$ is bounded. Then the operator $M_{z}$ is hyponormal if and only if

$$
\frac{a_{n}}{a_{n-1}} \geq \frac{a_{n+1}}{a_{n}} \quad \text { for all } n \geq 1
$$

(see Section 7 in [8], and note that the sequence $(\beta(n))$ there is related to $\left(a_{n}\right)$ via $\left.a_{n}=\beta(n)^{-2}\right)$. On the other hand, a sufficient condition for $\mathcal{H}$ being a complete Nevanlinna-Pick space is that the reverse inequalities

$$
\frac{a_{n}}{a_{n-1}} \leq \frac{a_{n+1}}{a_{n}} \quad \text { for all } n \geq 1
$$

hold (see Lemma 7.38 and Theorem 7.33 in [2]). Since this condition is not necessary, the two results do not immediately tell us anything new about weighted Hardy spaces satisfying both the Nevanlinna-Pick property and hyponormality of multiplication operators. Nevertheless, they seem to indicate that the presence of both properties is special.

The aim of this note is to show that the Hardy space is essentially the only complete Nevanlinna-Pick space whose multiplication operators are hyponormal. Recall that a reproducing kernel Hilbert space $\mathcal{H}$ with kernel $K$ on a set $X$ is called irreducible if $K(x, y)$ is never zero for $x, y \in X$ and if $K(\cdot, x)$ and $K(\cdot, y)$ are linearly independent for different $x, y \in X$. We call a set $A \subset \mathbb{D}$ a set of uniqueness for $H^{2}$ if the only element of $H^{2}$ which vanishes on $A$ is the zero function. The main result now reads as follows.

Theorem 1.1. Let $\mathcal{H}$ be an irreducible complete Nevanlinna-Pick space on a set $X$ with kernel $K$ such that all multiplication operators on $\mathcal{H}$ are hyponormal. Then one of the following possibilities holds:

(1) $X$ is a singleton and $\mathcal{H}=\mathbb{C}$.

(2) There is a set of uniqueness $A \subset \mathbb{D}$ for $H^{2}$, a bijection $j: X \rightarrow A$ and a nowhere vanishing function $\delta: X \rightarrow \mathbb{C}$ such that

$$
K(\lambda, \mu)=\delta(\lambda) \overline{\delta(\mu)} k(j(\lambda), j(\mu))
$$

where $k(z, w)=(1-z \bar{w})^{-1}$ denotes the Szegó kernel. Hence,

$$
H^{2} \rightarrow \mathcal{H}, \quad f \mapsto \delta(f \circ j),
$$

is a unitary operator. If $X$ is endowed with a topology such that $K$ is separately continuous on $X \times X$, then $j$ is continuous. If $X \subset \mathbb{C}^{n}$ and $K$ is holomorphic in the first variable, then $j$ is holomorphic. 
Since the Hardy space $H^{2}$ is a complete Nevanlinna-Pick space whose multiplication operators are hyponormal, it is easy to see that the same is true for every space as in part (2). Hence, this result characterizes Hilbert function spaces with these two properties.

Remark 1.2. (a) It is well known that sets of uniqueness for $H^{2}$ are characterized by the Blaschke condition (see, for example, [7, Section II 2]): A set $A \subset \mathbb{D}$ is a set of uniqueness for $H^{2}$ if and only if

$$
\sum_{a \in A}(1-|a|)=\infty .
$$

(b) The condition that $K(x, y)$ is never zero is not very restrictive. Indeed, if we drop this condition, then $X$ can be partitioned into sets $\left(X_{i}\right)$ such that the restriction of $\mathcal{H}$ to each $X_{i}$ (compare the next section) is an irreducible complete Nevanlinna-Pick space (see [2, Lemma 7.2]). This yields a decomposition of $\mathcal{H}$ into an orthogonal direct sum of irreducible complete Nevanlinna-Pick spaces $\mathcal{H}_{i}$. It is not hard to see that this decomposition is reducing for multiplication operators. Hence, all multiplication operators on $\mathcal{H}$ are hyponormal if and only if this is true for each summand. We omit the details.

Before we come to the proof of the main result, let us consider an application to Hilbert function spaces in higher dimensions. In particular, this applies to holomorphic Hilbert function spaces on the open unit ball in $\mathbb{C}^{n}$ for $n \geq 2$. Standard examples of such spaces either have the property that all multiplication operators are hyponormal (such as Hardy and Bergman space) or have the Nevanlinna-Pick property (such as the Drury-Arveson space, see the next section), but not both. This is not a coincidence.

Corollary 1.3. Let $n \geq 3$ be a natural number, and let $U \subset \mathbb{R}^{n}$ be an open set. Then there is no irreducible complete Nevanlinna-Pick space on $U$ which consists of continuous functions and whose multiplication operators are all hyponormal.

Proof. Assume toward a contradiction that $\mathcal{H}$ is such a Hilbert functions space, and let $K$ be its kernel. Since the functions in $\mathcal{H}$ are continuous, it follows that $K$ is separately continuous. Hence, Theorem 1.1 implies that there is a continuous injection $j: U \rightarrow \mathbb{D}$. But this is impossible if $n \geq 3$ due to Brouwer's domain invariance theorem [6].

\section{Embedding into Drury-Arveson space}

As a first step in the proof of the main result, we will embed the complete Nevanlinna-Pick space $\mathcal{H}$ into the Drury-Arveson space. Given a cardinal $d$, we write $\mathbb{B}_{d}$ for the open unit ball in $\ell^{2}(d)$. The Drury-Arveson space $H_{d}^{2}$ is the reproducing kernel Hilbert space on $\mathbb{B}_{d}$ with kernel

$$
k_{d}(z, w)=\frac{1}{1-\langle z, w\rangle} .
$$


If $d=1$, this is the Hardy space $H^{2}$. For $d \geq 2$, Arveson [4] exhibited multipliers on $H_{d}^{2}$ which are not hyponormal by showing that their spectral radius is strictly less than their multiplier norm. Indeed, if $z_{1}$ and $z_{2}$ denote the coordinate functions on $\mathbb{C}^{2}$, then $M_{z_{1} z_{2}}$ is not hyponormal on $H_{2}^{2}$, as

$$
\left\|M_{z_{1} z_{2}} z_{1} z_{2}\right\|^{2}=\frac{1}{6}<\frac{1}{4}=\left\|M_{z_{1} z_{2}}^{*} z_{1} z_{2}\right\|^{2}
$$

(see 4, Lemma 3.8]). This observation readily generalizes to $d \geq 2$.

Given a subset $Y \subset \mathbb{B}_{d}$, we write $\left.H_{d}^{2}\right|_{Y}$ for the reproducing kernel Hilbert space on $Y$ with kernel $\left.k_{d}\right|_{Y \times Y}$. A well-known result about Hilbert function spaces asserts that

$$
\left.H_{d}^{2}\right|_{Y}=\left\{\left.f\right|_{Y}: f \in H_{d}^{2}\right\},
$$

and that the restriction map $\left.f \mapsto f\right|_{Y}$ is a coisometry. Hence, if

$$
I(Y)=\left\{f \in H_{d}^{2}:\left.f\right|_{Y}=0\right\}
$$

denotes the kernel of the restriction map, then

$$
\left.H_{d}^{2} \ominus I(Y) \rightarrow H_{d}^{2}\right|_{Y},\left.\quad f \mapsto f\right|_{Y},
$$

is a unitary. The following theorem due to Agler and McCarthy provides the desired embedding of $\mathcal{H}$ into the Drury-Arveson space.

Theorem 2.1. Let $\mathcal{H}$ be an irreducible complete Nevanlinna-Pick space on a set $X$ with kernel $K$. Assume that $K$ is normalized at $\lambda_{0} \in X$ in the sense that $K\left(\lambda_{0}, \mu\right)=1$ for all $\mu \in X$. Then there is a cardinal $d$ and an injection $b: X \rightarrow \mathbb{B}_{d}$ with $b\left(\lambda_{0}\right)=0$ such that

$$
K(\lambda, \mu)=\frac{1}{1-\langle b(\lambda), b(\mu)\rangle} \quad(\lambda, \mu \in X) .
$$

Hence,

$$
H_{d}^{2} \ominus I(Y) \rightarrow \mathcal{H}, \quad f \mapsto\left(\left.f\right|_{Y}\right) \circ b,
$$

is a unitary operator, where $Y=b(X)$.

Proof. See [1], Theorem 3.1 in [5], or Theorem 8.2 and Theorem 7.31 in [2]. To deduce the second part from the first one, note that the identity for the kernels implies that

$$
\left.H_{d}^{2}\right|_{Y} \rightarrow \mathcal{H}, \quad f \mapsto f \circ b
$$

is unitary. Therefore, the composition of this map with the unitary operator in (11) is unitary as well.

In the above setting, let $\mathcal{F}_{Y}=H_{d}^{2} \ominus I(Y)$. This space is co-invariant under multiplication operators. Clearly, every $\varphi \in \operatorname{Mult}\left(H_{d}^{2}\right)$ restricts to a multiplier on $\left.H_{d}^{2}\right|_{Y}$, and hence gives rise to the multiplier $\left(\left.\varphi\right|_{Y}\right) \circ b$ on $\mathcal{H}$. If $U$ denotes the unitary operator in Theorem 2.1 , then

$$
U^{*} M_{\left(\left.\varphi\right|_{Y} \circ b\right)} U=\left.P_{\mathcal{F}_{Y}} M_{\varphi}\right|_{\mathcal{F}_{Y}} .
$$

Thus, if we assume that all multiplication operators on $\mathcal{H}$ are hyponormal, then all operators appearing on the right-hand side of the last identity are 
hyponormal as well. We will use this fact to show that $\mathcal{F}_{Y}$ can be identified with $H^{2}$.

\section{Proof of the MAIN Result}

The discussion at the end of the last section suggests studying compressions of multiplication operators to co-invariant subspaces such that the compressed operator is hyponormal. We need the following simple observation.

Lemma 3.1. Let $\mathcal{H}$ be a Hilbert space, let $T \in \mathcal{B}(\mathcal{H})$ and let $M \subset \mathcal{H}$ be a co-invariant subspace for $T$. Suppose that the compression of $T$ to $M$ is hyponormal. If $f \in M$ with $\left\|T^{*} f\right\|=\|T f\|$, then $T f \in M$.

Proof. Since $M$ is co-invariant under $T$, and since $\left.P_{M} T\right|_{M}$ is hyponormal, we have

$$
\left\|T^{*} f\right\| \leq\left\|P_{M} T f\right\| \leq\|T f\|=\left\|T^{*} f\right\|
$$

Consequently, $\left\|P_{M} T f\right\|=\|T f\|$, and hence $T f \in M$.

We will apply this observation to multiplication operators on $H_{d}^{2}$. Since the coordinate functions $z_{i}$ are multipliers on $H_{d}^{2}$, it follows from unitary invariance of the Drury-Arveson space that all functions of the form $\langle\cdot, w\rangle$ for $w \in \ell^{2}(d)$ are multipliers on $H_{d}^{2}$.

Lemma 3.2. Suppose that $\mathcal{F} \subset H_{d}^{2}$ is a closed subspace which is co-invariant under multiplication operators. Let $z \in \mathbb{B}_{d}$, and suppose that the compression $\left.P_{\mathcal{F}} M_{\langle\cdot, z\rangle}\right|_{\mathcal{F}}$ is hyponormal. Then the following assertions hold.

(a) If $1 \in \mathcal{F}$ and $K(\cdot, z) \in \mathcal{F}$, then $\langle\cdot, z\rangle \in \mathcal{F}$.

(b) If $\langle\cdot, z\rangle \in \mathcal{F}$, then $\langle\cdot, z\rangle^{n} \in \mathcal{F}$ for all $n \geq 1$.

Proof. (a) Clearly, we may assume that $z \neq 0$, and define $w=z /\|z\|$. Then

$$
\iota: H^{2} \rightarrow H_{d}^{2}, \quad \sum_{n=0}^{\infty} a_{n} \zeta^{n} \mapsto \sum_{n=0}^{\infty} a_{n}\langle\cdot, w\rangle^{n},
$$

is an isometry, where $\zeta$ denotes the identity function on $\mathbb{C}$. Under this embedding, the unilateral shift $M_{\zeta}$ on $H^{2}$ corresponds to the restriction of $M_{\langle\cdot, w\rangle}$ to the reducing subspace $\iota\left(H^{2}\right)$. In particular, $\left.M_{\langle\cdot, w\rangle}\right|_{\iota\left(H^{2}\right)}$ is an isometry.

Now, consider

$$
f=K(\cdot, z)-1=\sum_{n=1}^{\infty}\langle\cdot, z\rangle^{n} \in \mathcal{F} .
$$

Observe that $f$ is contained in the range of the isometry $\left.M_{\langle\cdot, w\rangle}\right|_{\iota\left(H^{2}\right)}$, hence

$$
\left\|M_{\langle\cdot, w\rangle} f\right\|=\|f\|=\left\|M_{\langle\cdot, w\rangle}^{*} f\right\| .
$$

Lemma 3.1 implies that $\mathcal{F}$ contains the element $M_{\langle\cdot, z\rangle} f$, and thus also

$$
f-M_{\langle\cdot, z\rangle} f=\langle\cdot, z\rangle \in \mathcal{F} \text {. }
$$


(b) The proof is by induction on $n$. The base case $n=1$ holds by assumption. Suppose that $n \geq 2$ and the assertion is true for $n-1$. The same argument as in the proof of part (a), applied to $\langle\cdot, z\rangle^{n-1}$ in place of $f$, shows that

so that

$$
\left\|M_{\langle\cdot, z\rangle}\langle\cdot, z\rangle^{n-1}\right\|=\left\|M_{\langle\cdot, z\rangle}^{*}\langle\cdot, z\rangle^{n-1}\right\|,
$$

by Lemma 3.1 .

$$
\langle\cdot, z\rangle^{n}=M_{\langle\cdot, z\rangle}\langle\cdot, z\rangle^{n-1} \in \mathcal{F}
$$

Given $Y \subset \mathbb{B}_{d}$, it can happen that there is a larger set $Z \supset Y$ such that every function in $\left.H_{d}^{2}\right|_{Y}$ extends uniquely to a function in $\left.H_{d}^{2}\right|_{Z}$. To account for that, we define

$$
\bar{Y}=\left\{z \in \mathbb{B}_{d}: f(z)=0 \text { for all } f \in I(Y)\right\} .
$$

Then $\bar{Y}$ is the largest set which contains $Y$ and satisfies this extension property. Moreover, it is easy to see that

$$
\bar{Y}=\left\{z \in \mathbb{B}_{d}: K(\cdot, z) \in H_{d}^{2} \ominus I(Y)\right\} .
$$

Lemma 3.3. Let $Y \subset \mathbb{B}_{d}$ be a set with $0 \in Y$, and set $\mathcal{F}_{Y}=H_{d}^{2} \ominus I(Y)$. If the compression $\left.P_{\mathcal{F}_{Y}} M_{\langle\cdot, w\rangle}\right|_{\mathcal{F}_{Y}}$ is hyponormal for every $w \in \mathbb{B}_{d}$, then $\bar{Y}$ is a complex ball, that is,

$$
\bar{Y}=M \cap \mathbb{B}_{d}
$$

for some closed subspace $M$ of $\ell^{2}(d)$.

Proof. Let $M$ be the closed linear span of $\bar{Y}$. Observe that for all $w \in \bar{Y}$, we have $K(\cdot, w) \in \mathcal{F}_{Y}$. Since $1=K(\cdot, 0) \in \mathcal{F}_{Y}$, part (a) of Lemma 3.2 implies that $\langle\cdot, w\rangle \in \mathcal{F}_{Y}$ for all $w \in \bar{Y}$. It follows that

$$
\langle\cdot, v\rangle \in \mathcal{F}_{Y} \quad \text { for all } v \in M
$$

as $v \mapsto\langle\cdot, v\rangle$ is a conjugate linear isometry. Using part (b) of Lemma 3.2. we deduce that

$$
K(\cdot, v)=\sum_{n=0}^{\infty}\langle\cdot, v\rangle^{n} \in \mathcal{F}_{Y}
$$

for all $v \in M \cap \mathbb{B}_{d}$. This argument shows that $\bar{Y} \supset M \cap \mathbb{B}_{d}$, and the reverse inclusion is trivial.

We can now prove the main result.

Proof of Theorem 1.1. If $X$ is a singleton, there is nothing to prove. Otherwise, fix $\lambda_{0} \in X$. Since $K$ is an irreducible kernel, it is nowhere zero, so we can consider the normalized kernel defined by

$$
\widetilde{K}(\lambda, \mu)=\frac{K(\lambda, \mu)}{\delta(\lambda) \overline{\delta(\mu)}}
$$

where

$$
\delta(\lambda)=\frac{K\left(\lambda, \lambda_{0}\right)}{\sqrt{K\left(\lambda_{0}, \lambda_{0}\right)}} .
$$


Then $\widetilde{K}\left(\lambda_{0}, \mu\right)=1$ for all $\mu \in X$. Moreover, if $\widetilde{\mathcal{H}}$ denotes the reproducing kernel Hilbert space with kernel $\widetilde{K}$, then

$$
\widetilde{\mathcal{H}} \rightarrow \mathcal{H}, \quad f \mapsto \delta f
$$

is a unitary operator. It is easy to see that $\widetilde{\mathcal{H}}$ also satisfies the hypotheses of Theorem 1.1. so we will work with $\widetilde{\mathcal{H}}$ instead of $\mathcal{H}$.

We will show that $\widetilde{\mathcal{H}}$ can be identified with $H_{d^{\prime}}^{2}$ for a suitable cardinal $d^{\prime}$. It will then follow that $d^{\prime}$ is necessarily 1. By Theorem 2.1, there is an injection $b: X \rightarrow \mathbb{B}_{d}$ for some cardinal $d$ such that $0=b\left(\lambda_{0}\right) \in b(X)$ and such that

$$
\widetilde{K}(\lambda, \mu)=k_{d}(b(\lambda), b(\mu))
$$

holds for all $\lambda, \mu \in X$. Define $Y=b(X)$ and $\mathcal{F}_{Y}=H_{d}^{2} \ominus I(Y)$, and note that $0 \in Y$. The discussion at the end of Section 2 now shows that $\mathcal{F}_{Y}$ satisfies the hypotheses of Lemma 3.3, hence

$$
\bar{Y}=M \cap \mathbb{B}_{d}
$$

for some closed subspace $M$. Let $d^{\prime}$ be the dimension of the Hilbert space $M$. As $X$ is not a singleton, $d^{\prime} \neq 0$. Clearly, $\mathcal{F}_{Y}=\mathcal{F}_{\bar{Y}}$, so that the restriction map from $\mathcal{F}_{Y}$ into $\left.H_{d}^{2}\right|_{\bar{Y}}$ is unitary. If $V$ is an isometry from $\ell^{2}\left(d^{\prime}\right)$ onto $M \subset \ell^{2}(d)$, we have

$$
k_{d}(V(z), V(w))=k_{d^{\prime}}(z, w) \quad \text { for all } z, w \in \mathbb{B}_{d^{\prime}} .
$$

Therefore,

$$
\mathcal{F}_{Y} \rightarrow H_{d^{\prime}}^{2}, \quad f \mapsto f \circ V,
$$

is a unitary operator as well. Combining this map with the unitary from Theorem 2.1, we obtain a unitary

$$
H_{d^{\prime}}^{2} \rightarrow \widetilde{\mathcal{H}}, \quad f \mapsto f \circ j
$$

where $j=V^{*} \circ b$.

By assumption, all multiplication operators on $\widetilde{\mathcal{H}}$ are hyponormal, hence the same is true for $H_{d^{\prime}}^{2}$. This is only possible if $d^{\prime}=1$ (see the discussion at the beginning of Section 2), so that the last operator is in fact a unitary from $H^{2}$ onto $\widetilde{\mathcal{H}}$. Injectivity of this operator implies that $A=j(X)$ is a set of uniqueness for $H^{2}$. Combining the identities for the various kernels, we see that

$$
K(\lambda, \mu)=\delta(\lambda) \overline{\delta(\mu)} k(j(\lambda), j(\mu)) \quad \text { for all } \lambda, \mu \in X,
$$

as asserted.

To prove the additional assertion, let $\lambda_{0} \neq \mu \in X$. Then $j(\mu) \neq 0$, so rearranging equation (2), we obtain for $j$ the formula

$$
j(\lambda)=(\overline{j(\mu)})^{-1}\left(1-\frac{\delta(\lambda) \overline{\delta(\mu)}}{K(\lambda, \mu)}\right) .
$$

Taking the definition of $\delta$ into account, it follows that $j$ is continuous (respectively holomorphic) whenever $K(\cdot, \mu)$ is. 
Remark 3.4. (a) Since $d^{\prime}=1$ in the last proof, the isometry $V$ is of the form $\lambda \mapsto \lambda w$ for some unit vector $w$ in the one-dimensional space $M$. It is easy to see that in this situation, the inverse of the unitary

$$
\mathcal{F}_{Y}=\mathcal{F}_{\bar{Y}} \rightarrow H^{2}, \quad f \mapsto f \circ V
$$

is given by

$$
H^{2} \rightarrow \mathcal{F}_{Y} \subset H_{d}^{2}, \quad \sum_{n=0}^{\infty} a_{n} \zeta^{n} \mapsto \sum_{n=0}^{\infty} a_{n}\langle\cdot, w\rangle^{n} .
$$

An isometric embedding of this type was used in the proof of Lemma 3.2 .

(b) For the most part of the proof of Theorem 1.1, we only used hyponormality of operators of the form $\left.P_{\mathcal{F}_{Y}} M_{\langle\cdot, w\rangle}\right|_{\mathcal{F}_{Y}}$ for $w \in \mathbb{B}_{d}$ (notation as above). If $\mathcal{H}$ is an irreducible complete Nevanlinna-Pick space with kernel $K$, normalized at some point $\lambda_{0}$, then these operators correspond to multiplication operators on $\mathcal{H}$ with multipliers of the form

$$
\varphi(\cdot)=\langle b(\cdot), w\rangle \quad\left(w \in \mathbb{B}_{d}\right),
$$

where $b$ is the injection from Theorem 2.1, These multipliers play the role of coordinate functions for Nevanlinna-Pick spaces (see the discussion preceding Beurling's theorem for Nevanlinna-Pick spaces [2, Theorem 8.67]).

The only argument which requires hyponormality of more general multiplication operators is the proof that $d^{\prime}=1$. Thus, if we weaken the hypothesis of Theorem 1.1 and only require hyponormality of multiplication operators corresponding to functions as in (3i), then $\mathcal{H}$ will be equivalent to $H_{d^{\prime}}^{2}$ (in the sense of part (2) of Theorem 1.1) for some cardinal $d^{\prime}$.

Acknowledgements. The author would like to thank his advisor, Ken Davidson, for his advice and support.

\section{REFERENCES}

1. Jim Agler and John E. McCarthy, Complete Nevanlinna-Pick kernels, J. Funct. Anal. 175 (2000), no. 1, 111-124.

2. _ Pick interpolation and Hilbert function spaces, Graduate Studies in Mathematics, vol. 44, American Mathematical Society, Providence, RI, 2002.

3. Călin-Grigore Ambrozie and Dan Timotin, On an intertwining lifting theorem for certain reproducing kernel Hilbert spaces, Integral Equations Operator Theory 42 (2002), no. $4,373-384$.

4. William Arveson, Subalgebras of $C^{*}$-algebras. III. Multivariable operator theory, Acta Math. 181 (1998), no. 2, 159-228.

5. Joseph A. Ball, Tavan T. Trent, and Victor Vinnikov, Interpolation and commutant lifting for multipliers on reproducing kernel Hilbert spaces, Operator theory and analysis (Amsterdam, 1997), Oper. Theory Adv. Appl., vol. 122, Birkhäuser, Basel, 2001, pp. $89-138$.

6. L. E. J. Brouwer, Beweis der Invarianz des n-dimensionalen Gebiets, Math. Ann. 71 (1911), no. 3, 305-313.

7. John B. Garnett, Bounded analytic functions, first ed., Graduate Texts in Mathematics, vol. 236, Springer, New York, 2007. 
8. Allen L. Shields, Weighted shift operators and analytic function theory, Topics in operator theory, Amer. Math. Soc., Providence, R.I., 1974, pp. 49-128. Math. Surveys, No. 13 .

Department of Pure Mathematics, University of Waterloo, Waterloo, ON N2L 3G1, CANADA

E-mail address: mphartz@uwaterloo.ca 